\section{BRAZIULIAN JOURNAL \\ OF MEDICAL AND BIOLOGICAL RESF.ARCH}

www.bjournal.com.br
ISSN 0100-879X

Volume 43 (01) 1-123 January 2010

BIOMEDICAL SCIENCES

AND

CLINICAL INVESTIGATION

Braz J Med Biol Res, J anuary 2010, Volume 43(1) 85-95

Lesion of the subthalamic nucleus reverses motor deficits but not death of nigrostriatal dopaminergic neurons in a rat 6-hydroxydopamine-lesion model of Parkinson's disease

V. Rizelio, R.E. Szawka, L.L. Xavier, M. Achaval, P. Rigon, L. Saur, F. Matheussi, A.M. Delattre, J.A. Anselmo-Franci, M. Meneses and A.C. Ferraz

The Brazilian Journal of Medical and Biological Research is partially financed by
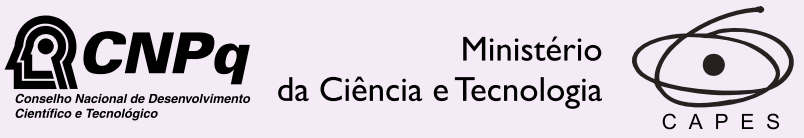

Ministério da Educação

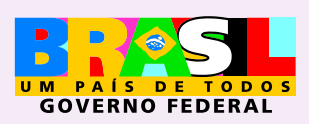




\title{
Lesion of the subthalamic nucleus reverses motor deficits but not death of nigrostriatal dopaminergic neurons in a rat 6-hydroxydopamine-lesion model of Parkinson's disease
}

\author{
V. Rizelio ${ }^{1}$, R.E. Szawka ${ }^{3}$, L.L. Xavier ${ }^{4}$, M. Achaval ${ }^{5}$, P. Rigon ${ }^{5}$, L. Saur ${ }^{5}$, \\ F. Matheussi ${ }^{1}$, A.M. Delattre ${ }^{1}$, J.A. Anselmo-Franci ${ }^{3}$, M. Meneses ${ }^{2}$ and A.C. Ferraz ${ }^{1}$ \\ ${ }^{1}$ Laboratório de Neurofisiologia, Departamento de Fisiologia, \\ 2Departamento de Anatomia, Universidade Federal do Paraná, Curitiba, PR, Brasil \\ ${ }^{3}$ Laboratório de Neuroendocrinologia, Departamento de Morfologia, Estomatologia e Fisiologia, \\ Faculdade de Odontologia de Ribeirão Preto, Universidade de São Paulo, Ribeirão Preto, SP, Brasil \\ ${ }^{4}$ Laboratório de Biologia Tecidual, Departamento de Ciências Morfofisiológicas, \\ Faculdade de Biociências, Pontifícia Universidade Católica do Rio Grande do Sul, Porto Alegre, RS, Brasil \\ ${ }^{5}$ Laboratório de Histofisiologia Comparada, Departamento de Ciências Morfológicas, \\ Instituto de Ciências Básicas da Saúde, Universidade Federal do Rio Grande do Sul, Porto Alegre, RS, Brasil
}

\begin{abstract}
The objective of the present study was to determine whether lesion of the subthalamic nucleus (STN) promoted by N-methylD-aspartate (NMDA) would rescue nigrostriatal dopaminergic neurons after unilateral 6-hydroxydopamine (6-OHDA) injection into the medial forebrain bundle (MFB). Initially, $16 \mu \mathrm{g}$ 6-OHDA (6-OHDA group) or vehicle (artificial cerebrospinal fluid - aCSF; Sham group) was infused into the right MFB of adult male Wistar rats. Fifteen days after surgery, the 6-OHDA and Sham groups were randomly subdivided and received ipsilateral injection of either $60 \mathrm{mM}$ NMDA or aCSF in the right STN. Additionally, a control group was not submitted to stereotaxic surgery. Five groups of rats were studied: 6-OHDA/NMDA, 6-OHDA/Sham, Sham/ NMDA, Sham/Sham, and control. Fourteen days after injection of 6-OHDA, rats were submitted to the rotational test induced by apomorphine $(0.1 \mathrm{mg} / \mathrm{kg}, i p)$ and to the open-field test. The same tests were performed again 14 days after NMDA-induced lesion of the STN. The STN lesion reduced the contralateral turns induced by apomorphine and blocked the progression of motor impairment in the open-field test in 6-OHDA-treated rats. However, lesion of the STN did not prevent the reduction of striatal concentrations of dopamine and metabolites or the number of nigrostriatal dopaminergic neurons after 6-OHDA lesion. Therefore, STN lesion is able to reverse motor deficits after severe 6-OHDA-induced lesion of the nigrostriatal pathway, but does not protect or rescue dopaminergic neurons in the substantia nigra pars compacta.
\end{abstract}

Key words: Subthalamic nucleus; Parkinson's disease; Substantia nigra pars compacta; 6-Hydroxydopamine;

Tyrosine hydroxylase immunohistochemistry

\section{Introduction}

Parkinson's disease (PD) is a progressive neurodegenerative disorder that results from a loss of dopaminergic neurons in the substantia nigra pars compacta (SNpc) (1). The degeneration of mesencephalic dopaminergic cells causes significant dopamine (DA) depletion in the corpus striatum, leading to debilitating motor dysfunction when DA reduction is greater than $80 \%$ (2). PD symptoms include akinesia, rigidity, resting tremor, slow movement, gait dysfunction, and postural instability (3).

The striatal DA deficit leads to the disinhibition of the indirect pathway of the basal ganglia circuit. As a consequence, the subthalamic nucleus (STN) becomes hyper- 
active in PD $(4,5)$, increasing the excitatory drive of the output of basal ganglia nuclei and thereby increasing the inhibition of thalamocortical neurons (6). Moreover, it has been suggested that STN hyperactivity increases the deterioration of existing nigrostriatal dopaminergic neurons through excessive glutamatergic stimulation, resulting in N-methyl-D-aspartate (NMDA)-mediated excitotoxicity and further neuronal loss in PD $(3,7,8)$. This is supported by clinical studies showing that alteration in STN activity by high-frequency stimulation (HFS) improves motor symptoms in PD (9).

Thus, it is possible that STN inactivation may have a neuroprotective action on the SNpc dopaminergic cells, reducing the loss of dopaminergic neurons and decreasing motor impairments in PD $(7,10,11)$. In experimental models of PD induced by parkinsonian neurotoxins such as 6-hydroxydopamine (6-OHDA) or 1-methyl-2-phenyl1,2,3,6-tetrahydropyridine (MPTP), inhibition of STN function by ablation $(12-15)$, HFS $(16,17)$ or pharmacological antagonism $(18,19)$ has been reported to ameliorate some of the parkinsonian motor deficits and even to protect nigrostriatal dopaminergic neurons against the injury induced by these neurotoxins. On the other hand, some investigators were unable to find any neuroprotective effects of STN inactivation on SNpc neuron survival (2022). For this reason, it remains to be determined which circuitry is altered in the basal ganglia when glutamatergic pathways are suppressed and motor improvements take place. It is also important to determine whether the increase in striatal DA metabolism is due to an effect on the survival of SNpc neurons or if it reflects an altered activity of another pathway in the basal ganglia, and also if the increase in striatal DA levels is responsible for the motor improvement seen in Parkinsonism.

Two protocols are generally used to study glutamatergic lesion of the STN. One evaluates the possible neuroprotection offered by the lesion and the other, the recovery of damaged dopaminergic neurons. In the former protocol, the STN lesion is made prior to injecting the neurotoxin, while in the latter the STN lesion is performed after injecting it. PD symptoms appear when at least $60 \%$ of dopaminergic neurons are lost (23). Because the rescue protocol is likely to represent more closely the clinical condition, the objective of the present study was to determine whether STN lesion would rescue nigrostriatal dopaminergic neurons after 6-OHDA injection into the medial forebrain bundle (MFB). We therefore examined the effect of glutamatergic input ablation, promoted by NMDA-induced lesion of the STN, on the nigrostriatal DA system, as determined by DA and metabolite concentrations in the striatum and tyrosine hydroxylase (TH)-immunoreactivity in the SNpc. In addition, in order to confirm the effects of STN ablation on parkinsonian motor deficits, behavioral tests of motor function were also conducted.

\section{Material and Methods}

\section{Animals}

Adult male Wistar rats from our own breeding stock weighing $300-400 \mathrm{~g}$ at the beginning of the experiments were used. The animals were maintained in a temperaturecontrolled room $\left(22 \pm 2^{\circ} \mathrm{C}\right)$ on a $12 / 12$-h light cycle (lights on at 7:00 am) and had free access to food and water. All studies involving the animals strictly followed the Guide for the Care and Use of Experimental Animals (Canadian Council on Animal Care) and were approved by the Federal University of Paraná Committee of Animal Welfare. Efforts were made to minimize animal use and their suffering in these experiments.

\section{Experimental design}

Rats received two stereotaxic surgical interventions in the right side of the brain. First, $16 \mu \mathrm{g}$ 6-OHDA (6-OHDA group) or vehicle, artificial cerebrospinal fluid (aCSF; Sham group), was infused into the right MFB. The control group was not submitted to stereotaxic surgery. Fifteen days after the first surgery, the 6-OHDA and Sham groups received an ipsilateral injection of $60 \mathrm{mM}$ NMDA (6-OHDA/NMDA and Sham/NMDA groups) or aCSF in the right STN (6-OHDA/ Sham and Sham/Sham groups). Accordingly, 5 groups containing a minimum of 7 rats each, unless otherwise stated, were studied: control, Sham/Sham, 6-OHDA/Sham, Sham/NMDA, and 6-OHDA/NMDA. Fourteen days after the first surgery, rats underwent a behavioral test for locomotor activity, followed $2 \mathrm{~h}$ later by the drug-induced rotation behavior (first test). The same tests were performed again 14 days after the second surgery (second test). For neurochemical evaluation, rats were killed by decapitation 2 days after the second behavioral test. Brains were removed and the striatum tissue was dissected and assayed for DA and its metabolites, 3,4-dihydroxyphenylacetic acid (DOPAC) and 4-hydroxy-3-methoxy-phenylacetic acid (HVA). For $\mathrm{TH}$ immunostaining, rats from the Sham/Sham, Sham/ NMDA, 6-OHDA/Sham, and 6-OHDA/NMDA groups were anesthetized and transcardially perfused and the brains were removed and processed for immunohistochemistry. The absorbance of $\mathrm{TH}$ staining and the number of $\mathrm{TH}$ immunoreactive ( $\mathrm{TH}$-ir) neurons were evaluated in the SNpc and ventral tegmental area (VTA). In this analysis, control groups were not studied since we had previously demonstrated that $\mathrm{TH}$-immunoreactivity is similar in control and Sham-operated rats $(24,25)$. The number of rats studied in each experimental group was 4 .

\section{Stereotaxic surgeries}

Rats received atropine sulfate $(0.4 \mathrm{mg} / \mathrm{kg}$, ip) to suppress salivation and penicillin G-procaine $(5,000,000 \mathrm{U}$ in 5 $\mathrm{mL}, \mathrm{im}$ ), and were anesthetized ip with $3 \mathrm{~mL} / \mathrm{kg}$ equithesin (1\% thiopental, $4.25 \%$ chloral hydrate, $2.13 \%$ magnesium sulfate, $42.8 \%$ propylene glycol, and $3.7 \%$ ethanol in water). 
In the first surgery, 6-OHDA HCl $(16 \mu \mathrm{g}$ in $4 \mu \mathrm{L}$ aCSF with $0.2 \%$ ascorbate; Sigma, USA) was unilaterally infused $(0.5$ $\mu \mathrm{L} / \mathrm{min}$ ) into the right MFB through a 30 -gauge needle. In sham-operated rats, $4 \mu \mathrm{L}$ aCSF was infused into the MFB. The coordinates for the injections were: anteroposterior (AP), -1.9 mm from bregma, mediolateral (ML), -1.9 mm from the midline, dorsoventral (DV), $-7.2 \mathrm{~mm}$ from the skull, as adapted from Paxinos and Watson (26). In the second surgery, $1 \mu \mathrm{L} 60 \mathrm{mM}$ NMDA (Sigma) or aCSF was infused into the right STN with a 30 -gauge needle at $0.5 \mu \mathrm{L} / \mathrm{min}$. The coordinates used were: AP, $-3.8 \mathrm{~mm}$ from bregma, $\mathrm{ML}$, $-2.5 \mathrm{~mm}$ from midline, DV, $-8.1 \mathrm{~mm}$ from the skull, adapted from Paxinos and Watson (26). To avoid drug reflow, a 2-min interval was allowed to elapse between the completion of each infusion and withdrawal of the needle. The composition of aCSF was as follows: $0.15 \mathrm{M} \mathrm{NaCl}, 2.75 \mathrm{mM} \mathrm{KCl}$, $1.2 \mathrm{mM} \mathrm{CaCl}_{2}$, and $0.85 \mathrm{mM} \mathrm{MgCl}$.

\section{Locomotor activity}

Hypokinesia was evaluated using an open field, which consisted of a circular arena ( $1 \mathrm{~m}$ in diameter), with the floor divided into 19 sections, limited by a $40-\mathrm{cm}$ circular wall. This arena was illuminated by four $60-\mathrm{W}$ lights and placed in a sound-attenuated temperature-controlled illuminated room. Each rat was placed alone in the center of the arena and observed while exploring the open field for $5 \mathrm{~min}$. The total number of squares crossed and rearing activity were determined over a period of 5 min (27). Before placing another animal, the open field was cleaned with $10 \%$ ethanol to attenuate odors. All groups were tested for locomotor activity 14 days after the first surgery (test 1 ) and 14 days after the second surgery (test 2).

\section{Drug-induced rotational behavior}

Motor asymmetry following unilateral lesion of the nigrostriatal pathway was assessed by apomorphine-induced rotational behavior. The rats were injected subcutaneously with $0.1 \mathrm{mg} / \mathrm{kg}$ apomorphine hydrochloride (Sigma) dissolved in $0.9 \% \mathrm{NaCl}$. Total turns contralateral to the lesion were counted over a period of $15 \mathrm{~min}$. All groups were tested for rotational behavior 14 days after the first surgery (test 1 ) and 14 days after the second surgery (test 2). Repeated stimulation with a DA agonist may produce an increase in DA-mediated behavioral responses (priming effect). Nevertheless, a two-week interval is probably enough time to avoid the priming effect of the first rotational test (12).

\section{Neurochemical assay}

Following decapitation, the brains were rapidly removed and the striatum tissues were dissected, weighed, immediately frozen on dry ice and stored at $-80^{\circ} \mathrm{C}$ until assayed for DA, DOPAC and HVA by high-performance liquid chromatography coupled with electrochemical detection (HPLC-ED), as previously described (25). The striatum tissues were homogenized in $800 \mu \mathrm{L}$ of a solution contain- ing 0.2 M perchloric acid (Merck, Germany), $0.1 \mathrm{mM}$ EDTA (Merck) and $0.45 \mu \mathrm{M}$ 3,4-dihydroxybenzylamine (DHBA; Aldrich, USA) as the internal standard. The homogenates were centrifuged for $20 \mathrm{~min}$ at $12,000 \mathrm{~g}$ and the supernatant was filtered through a 0.22- $\mu$ m filter (Millex PVDF, Millipore, USA). Ten microliters of each sample was injected with an auto injector (SIL-10Advp; Shimadzu, Japan) into an HPLCED system. Separation was performed on a 4.6 x 250-mm reversed-phase C18 column (Shim-pack VP-ODS, $5 \mu \mathrm{m}$; Shimadzu), preceded by a 4.6 x 10-mm C18 guard column (Shim-pack GVP-ODS, $5 \mu \mathrm{m}$; Shimadzu). The mobile phase consisted of $100 \mathrm{mM}$ sodium dihydrogen phosphate monohydrate, $10 \mathrm{mM}$ sodium chloride, $0.1 \mathrm{mM}$ EDTA, $0.40 \mathrm{mM}$ sodium 1-octanesulfonic acid (Sigma) and 20\% methanol (Omnisolv, EMD Chemical Inc., USA). pH was adjusted to 3.5 with phosphoric acid. Flow rate was maintained at 0.9 $\mathrm{mL} / \mathrm{min}$ (LC-10Advp; Shimadzu). The detector potential was $0.65 \mathrm{~V}$ vs in situ Ag/AgCl (Decade, VT-03 electrochemical flow cell; Antec Leyden, Netherlands). Chromatography data were plotted using Class-VP software (Shimadzu). DA, DOPAC, and HVA were identified based on their peak retention times. Quantification was performed by the internal standard method using DHBA as the internal standard based on the area under the peak. All samples were measured in the same analysis and the intra-assay coefficient of variation was less than $5 \%$ for all compounds measured. Data were normalized by tissue wet weight, and DA and metabolite concentrations in the lesioned striatum (right) are reported as a percentage of those on the contralateral side.

\section{Histological evaluation of STN lesion}

Cell loss in the STN and the correct location of NMDA injection were determined using two-dimensional images of Nissl-stained sections $(28,29)$.

\section{TH immunohistochemistry}

Rats were deeply anesthetized with $200 \mathrm{mg} / \mathrm{kg}$ sodium thiopental, ip, and transcardially perfused with saline followed by $4 \%$ paraformaldehyde in $0.1 \mathrm{M}$ phosphate buffer (PB), pH $7.4(24,25,28)$. After perfusion, the brains were removed from the skulls, postfixed in the same solution at room temperature for $2 \mathrm{~h}$ and cryoprotected by immersion in $30 \%$ sucrose solution in $\mathrm{PB}$ at $4^{\circ} \mathrm{C}$ until they sank. For each brain, serial coronal sections $(50 \mu \mathrm{m})$ were obtained with a cryostat (Leitz, Digital 1702 , Germany) at $-20^{\circ} \mathrm{C}$ and collected in PB. Free-floating sections were pretreated with $10 \%$ methanol diluted in $3 \%$ hydrogen peroxidase for 30 min, blocked with 3\% normal goat serum in $0.1 \mathrm{M} \mathrm{PB}$, $0.9 \% \mathrm{NaCl}, \mathrm{pH} 7.4$ (PBS) containing $0.3 \%$ Triton $\mathrm{X}-100$ (PBS-Tx, Sigma) for $30 \mathrm{~min}$ and incubated with mouse anti-TH antibody (Sigma) at 1:750 in 3\% normal goat serum in PBS-Tx for $48 \mathrm{~h}$ at $4^{\circ} \mathrm{C}$. Sections were then incubated with a biotinylated secondary antibody (Dako Corporation, USA) at 1:200 in PBS-Tx at room temperature for $2 \mathrm{~h}$, and with peroxidase-conjugated streptavidin (Dako) at 1:100 
in PBS for $90 \mathrm{~min}$ at room temperature. The antibodyperoxidase complex was developed by incubating the sections in a medium containing $0.06 \% 3,3$ diaminobenzidine (DAB, Sigma) dissolved in PBS for $10 \mathrm{~min}$, and then in the same solution containing $1 \mu \mathrm{M} 3 \% \mathrm{H}_{2} \mathrm{O}_{2}$ per $\mathrm{mL}$ of DAB medium for $10 \mathrm{~min}$. Next, the sections were rinsed with PBS, dehydrated with ethanol, cleared with xylene, covered with Permount, and coverslipped. Control sections were prepared by omitting the primary antibody and replacing it with PBS. The brains of all animals were fixed and postfixed for the same time in the same batch of solution rigorously processed at the same time, and the sections were incubated in an identical medium for the same period of time. This precaution was taken to avoid overreaction, differences in chromogen reaction, saturation of absorbance, and changes in background levels $(24,25,28,30)$. Coronal sections of the SNpc were selected according to the atlas of Paxinos and Watson (26) and the readings were obtained between the following coordinates: interaural $4.2 \mathrm{~mm}$, bregma $-4.8 \mathrm{~mm}$ and interaural $-2.7 \mathrm{~mm}$, bregma $-6.3 \mathrm{~mm}$. In the SNpc and the VTA, the absorbance of the $\mathrm{TH}$-ir neurons and the number of $\mathrm{TH}$-ir neurons $/ \mathrm{mm}^{2}$ were determined as previously described $(24,25)$. Briefly, images of coronal sections were digitalized and three equidistant

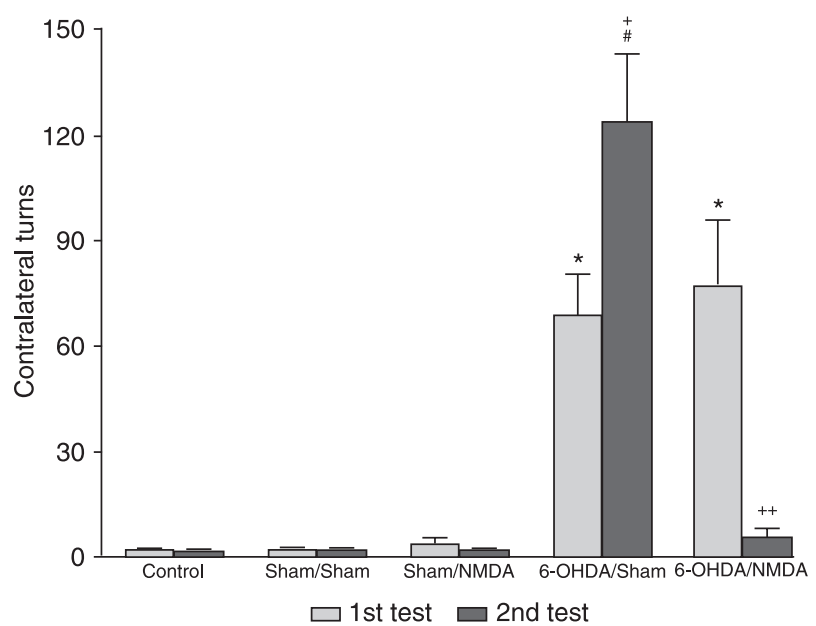

Figure 1. Total number of apomorphine-induced contralateral turns during a period of $15 \mathrm{~min}$. Data are reported as means \pm SEM for the following groups: control $(N=8)$, SNpc sham lesion/STN sham lesion (Sham/Sham; $N=7$ ), SNpc sham lesion/ STN NMDA lesion (Sham/NMDA; $\mathrm{N}=8$ ), SNpc 6-OHDA lesion/ STN sham lesion (6-OHDA/Sham; $N=10$ ), and SNpc 6-OHDA lesion/STN NMDA lesion (6-OHDA/NMDA; $N=10)$ in the first and second tests. SNpc = substantia nigra pars compacta; STN = subthalamic nucleus; NMDA = N-methyl-D-aspartate; 6-OHDA $=6$-hydroxydopamine. ${ }^{*} \mathrm{P}<0.01$ compared to the other groups in the first test; \#P $<0.01$ compared to the other groups in the second test (one-way ANOVA). ${ }^{+} \mathrm{P}<0.05$ compared to the first test in the same group. ${ }^{++} \mathrm{P}<0.01$ compared to the first test in the same group (Student $t$-test). areas of interest measuring $1200 \mu \mathrm{m}^{2}$ were overlaid onto the image to measure absorbance and the number of $\mathrm{TH}$-ir neurons $/ \mathrm{mm}^{2}$. At least 30 readings in each of 10 sections were obtained from each side of the brain.

Absorbance was calculated using the following formula:

$A(x, y)=-\log [($ intensity $(x, y)-$ black $) /($ incident - black $)]$

where: $A$ is absorbance, intensity $(x, y)$ is the intensity at pixel $(x, y)$, black is the intensity generated when no light goes through the material (5.3 in our case), and incident is the intensity of the incident light (252.4 in our case). To determine neuronal density, the sum of $\mathrm{TH}$-ir neurons located inside this square or intersected by the lower and/ or right edge of the square were counted. The neurons that were intersected by the upper and/or left edge of the square were not counted $(24,25,28)$.

Count and absorbance measurements were carried out by two specialists in histology blind to the source of the images. All procedures were done using a Nikon Eclipse E-600 (50X) microscope coupled to a Pro-Series High Performance CCD camera and Image Pro Plus Software 4.1 (Media Cybernetics, USA).

\section{Statistical analysis}

Data are reported as means \pm SEM. Behavioral and neurochemical data were analyzed by one-way analysis of variance (ANOVA) and TH immunohistochemical data by two-way and one-way ANOVA. In all analyses, ANOVA was followed by the Duncan post hoc test. Differences between the first and second behavioral tests were analyzed by the Student $t$-test for dependent samples. $P<0.05$ was considered to be statistically significant.

\section{Results}

\section{Drug-induced rotational behavior}

Contralateral turns induced by apomorphine were increased in the 6-OHDA groups in the first test compared to Sham and control groups $\left(F_{4,38}=12.29, \mathrm{P}<0.01\right)$, as shown in Figure 1 . In the second test, the number of contralateral turns observed in the 6-OHDA/Sham group was greater than all the other groups $\left(F_{4,38}=10.57, \mathrm{P}<\right.$ 0.01 ). When each group was analyzed considering the first vs the second test, the 6-OHDA/NMDA group showed a significant reduction in total contralateral turns $(t=3.37$, $P<0.01)$. On the other hand, the number of contralateral turns increased in the 6-OHDA/Sham group $(t=2.37, \mathrm{P}<$ $0.05)$. There were no significant differences between the first and second tests of the control, Sham/Sham or Sham/ NMDA groups.

\section{Locomotor activity}

Analysis of the total squares crossed in the open field 
(Figure 2A) showed that, in the first test, 6-OHDA animals exhibited reduced exploratory activity compared to control and Sham animals $\left(F_{4,47}=5.19, \mathrm{P}<0.01\right)$. In the second test, the 6-OHDA groups still crossed fewer squares compared to the control and Sham groups $\left(F_{4,47}=17.17\right.$, $\mathrm{P}<0.001)$; however, the reduction was attenuated in the 6-OHDA/NMDA group when compared to the 6-OHDA/ Sham group $(P<0.05)$. In the 6-OHDA/Sham group, there was a greater decrease in the number of squares crossed in the second test compared to the first $(t=5.92, \mathrm{P}<0.001)$. This further reduction was prevented by the STN lesion in the 6-OHDA/NMDA group ( $t=0.45, \mathrm{P}=0.66)$. There were no significant changes between the first and second tests in control, Sham/Sham and Sham/NMDA groups.

As illustrated in Figure 2B, evaluation of rearing movements in the open field showed results similar to those for the squares crossed. In the first test, 6-OHDA groups exhibited fewer rearing movements than control and Sham groups $\left(F_{4,47}=11.12, \mathrm{P}<0.001\right)$. In the second test, the 6-OHDA groups still exhibited decreased rearing activity compared to control and Sham groups $\left(F_{4,47}=11.99, \mathrm{P}<\right.$ $0.001)$. However, the reduction was smaller in the 6-OHDA/ NMDA group than in the 6-OHDA/Sham group $(P<0.05)$. In the 6-OHDA/Sham group, rearing activity was further reduced in the second test compared to the first one $(t=$ $4.89, \mathrm{P}<0.001)$. This further reduction was prevented by the STN lesion in the 6-OHDA/NMDA group $(\mathrm{t}=0.26, \mathrm{P}=$ $0.80)$. There were no significant changes between the first and second tests in the control, Sham/Sham and Sham/ NMDA groups.

\section{Histological evaluation of STN lesion}

The number of neurons in the STN was quantified in the right and the left hemisphere. NMDA injection promoted a significant lesion in the STN with a cell loss of approximately $84 \%$ in the right versus the left hemisphere, without involvement of adjacent nuclei (Figure 3).

\section{Neurochemical assay}

Analysis of DA, DOPAC, and HVA concentrations in the non-lesioned striatum (left side) revealed no significant differences between the control, Sham/Sham, Sham/NMDA, 6-OHDA/Sham, and 6-OHDA/NMDA groups. In the left striatum, the average DA, DOPAC, and HVA concentrations were $5.89 \pm 0.62,3.37 \pm 0.16$ and $0.67 \pm 0.03 \mathrm{ng} / \mathrm{mg}$ tissue (mean \pm SEM), respectively. These data validated the use of contralateral values as controls for the unilateral lesion of the nigrostriatal pathway. There was a significant decrease in striatal DA $\left(F_{4,28}=32.04, \mathrm{P}<0.001\right)$, DOPAC $\left(F_{4,28}=\right.$ $43.00, \mathrm{P}<0.001)$ and $\operatorname{HVA}\left(F_{4,28}=66.54, \mathrm{P}<0.001\right)$ concentrations in the groups treated with 6-OHDA compared to the other groups (Figure 4). On the other hand, the STN lesion in Sham and 6-OHDA-lesioned rats did not alter the striatal concentrations of DA $(P=0.99)$, DOPAC $(P=0.99)$ or HVA $(P=0.99)$ compared to Sham/Sham and 6-OHDA/ Sham rats, respectively.
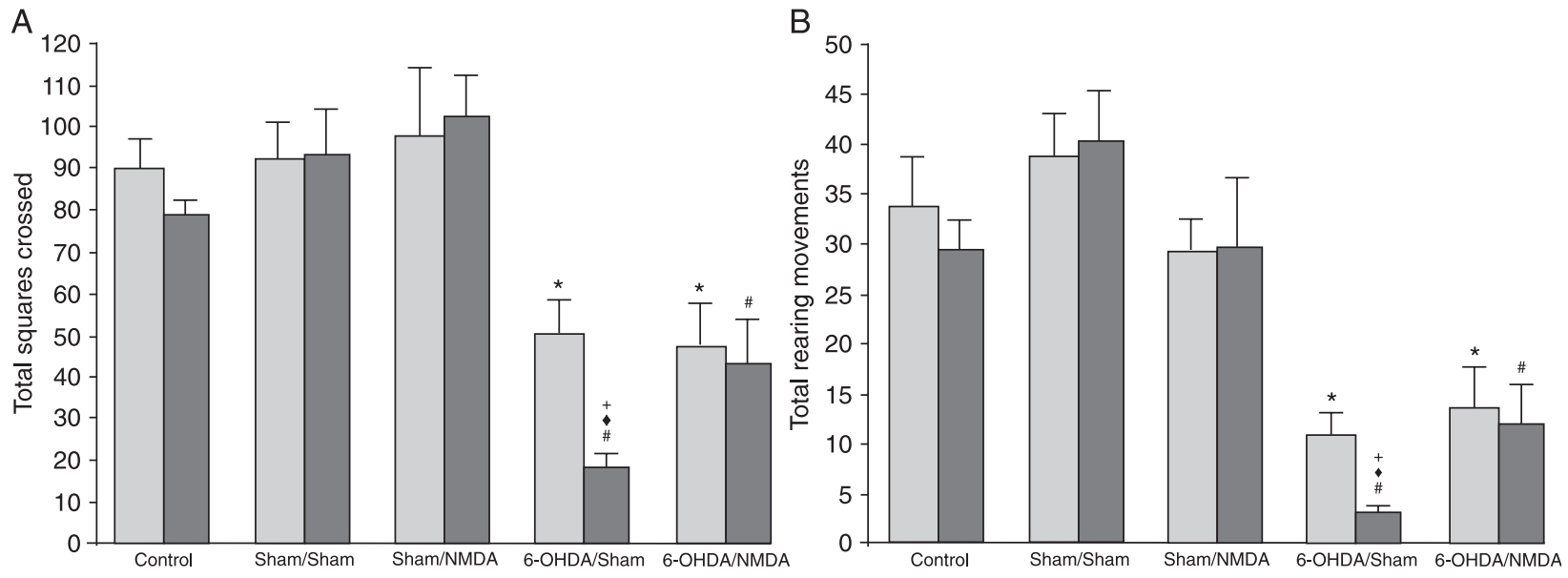

Figure 2. Open-field locomotor activity during a 5-min period. Data are reported as means \pm SEM for the following groups: control $(\mathrm{N}=10)$, SNpc sham lesion/STN sham lesion (Sham/Sham; $N=10)$, SNpc sham lesion/STN NMDA lesion (Sham/NMDA; $N=10)$, SNpc 6-OHDA lesion/STN sham lesion (6-OHDA/Sham; $\mathrm{N}=11$ ), and SNpc 6-OHDA lesion/STN NMDA lesion (6-OHDA/NMDA; N = 11 ) in the first and second tests. SNpc = substantia nigra pars compacta; STN = subthalamic nucleus; NMDA = N-methyl-D-aspartate; 6 -OHDA = 6-hydroxydopamine. $A$, Total squares crossed. ${ }^{*} \mathrm{P}<0.01$ compared to the other groups in the first test; ${ }^{\#} \mathrm{P}<0.001$ compared to the other groups in the second test; ${ }^{\bullet} \mathrm{P}<0.05$ compared to the 6-OHDA/NMDA group in the second test (one-way ANOVA). ${ }^{+} \mathrm{P}<$ 0.001 compared to the first test in the same group (Student $t$-test). $B$, Total number of rearing movements. ${ }^{*} P<0.001$ compared to the other groups in the first test; ${ }^{\#} P<0.001$ compared to the other groups in the second test; ${ }^{\bullet} P<0.05$ compared to the $6-O H D A / N M D A$ group in the second test (one-way ANOVA). ${ }^{+} \mathrm{P}<0.001$ compared to the first test in the same group (Student $t$-test). 


\section{TH immunohistochemistry}

Injection of 6-OHDAinto the MFB significantly decreased the absorbance of TH immunostaining $\left(F_{1,28}=92.34, \mathrm{P}<\right.$ $0.001)$ and the number of $\mathrm{TH}$-ir neurons $\left(F_{1,28}=32.71\right.$, $P<0.001$ ) in the SNpc, as illustrated in Figures 5 and $6 \mathrm{~A}$ and B. STN lesion did not protect the SNpc neurons from 6-OHDA-induced lesion, as determined by the equal decrease in absorbance $(P=1.00)$ or neuronal density $(\mathrm{P}=0.74)$ of $\mathrm{TH}$-ir neurons. In the VTA, there was also a 6 -OHDA-induced decrease in the absorbance of $\mathrm{TH}\left(F_{1,28}\right.$ $=29.99, \mathrm{P}<0.001)$ and number of $\mathrm{TH}$-ir neurons $\left(F_{1,28}=\right.$
29.61, $\mathrm{P}<0.001$; Figures 5 and $6 \mathrm{C}$ and D). As also observed for the SNpc, TH immunostaining did not differ between the 6-OHDA/NMDA and 6-OHDA/Sham groups.

\section{STN lesion and behavioral effects}

The effect of STN lesion in rats recovering from anesthesia following aCSF (Sham/NMDA group) or 6-OHDA (6OHDA/NMDA) infusion into the MFB was the manifestation of a marked contralateral hemiballismus. This response was transient, lasting approximately $16 \mathrm{~h}$. Temporary hemiballismus has also been described in rats awakening from
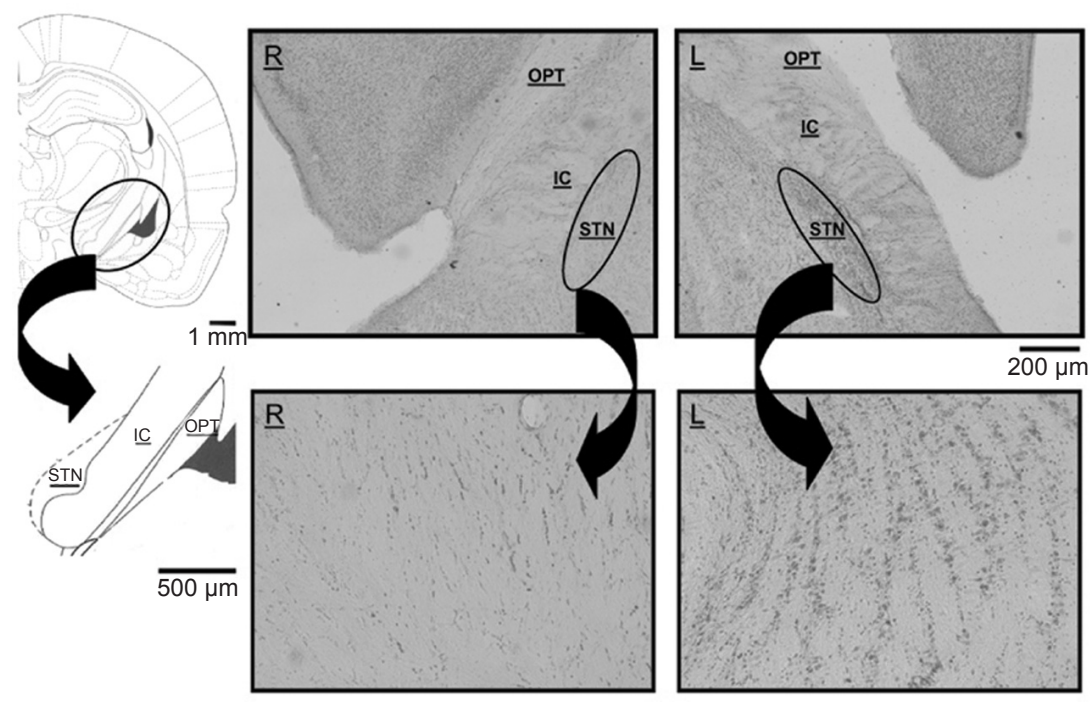

Figure 3. Digitized images of coronal sections of the subthalamic nucleus in the right $(R)$ and left $(L)$ hemispheres. Note a severe loss of neurons in the left STN after unilateral NMDA injection. The schematic drawings were modified from the Paxinos and Watson atlas (26). IC = internal capsule; OPT = optic tract; STN = subthalamic nucleus.

$\overline{100 \mu \mathrm{m}}$
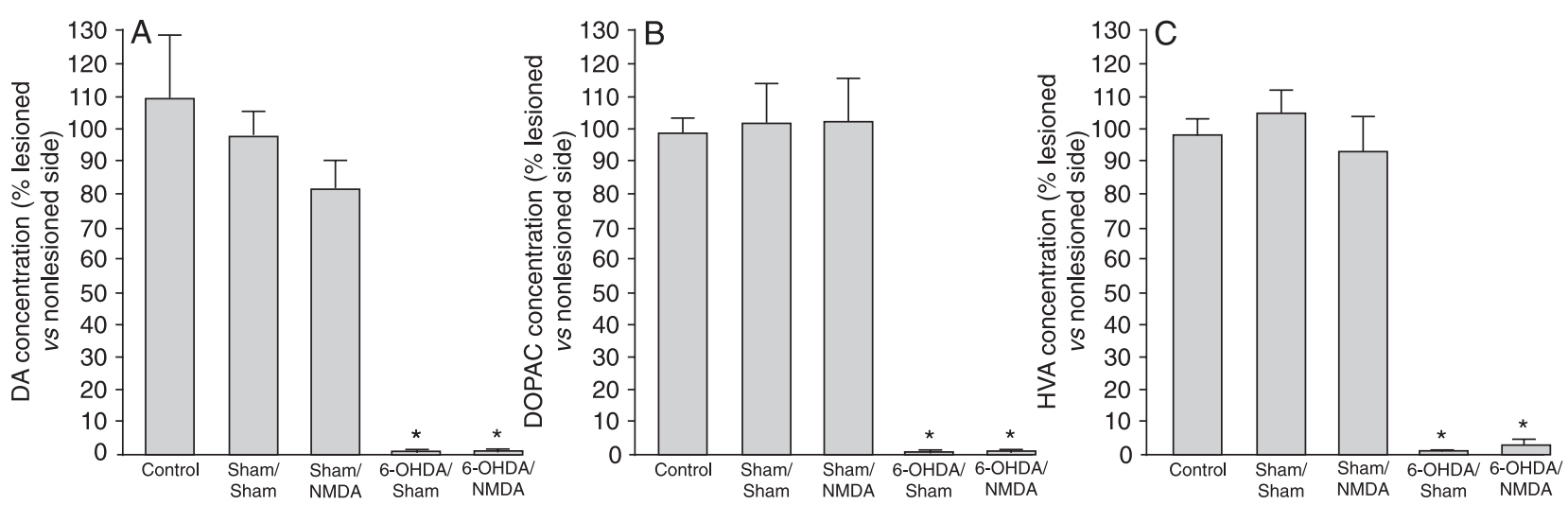

Figure 4. Neurochemical analysis of DA, DOPAC and HVA concentrations in the striatum. Percentage of DA, DOPAC and HVA levels remaining on the lesioned side (right) compared with the non-lesioned side of the following groups: control $(\mathrm{N}=6), \mathrm{SNpc}$ sham lesion/ STN sham lesion (Sham/Sham; $N=6$ ), SNpc sham lesion/STN NMDA lesion (Sham/NMDA; $N=7$ ), SNpc 6-OHDA lesion/STN sham lesion (6-OHDA/Sham; $\mathrm{N}=7$ ), and SNpc 6-OHDA lesion/STN NMDA lesion (6-OHDA/NMDA; $N=7$ ). Data are reported as means \pm SEM. DA = dopamine; DOPAC = 3,4-dihydroxyphenylacetic acid; HVA = 4-hydroxy-3-methoxy-phenylacetic acid; $\mathrm{SNpc}=$ substantia nigra pars compacta; STN = subthalamic nucleus; NMDA = N-methyl-D-aspartate; 6-OHDA $=6$-hydroxydopamine. ${ }^{*} \mathrm{P}<0.001 \mathrm{com}$ pared to control, Sham/Sham and Sham/NMDA groups (one-way ANOVA) 


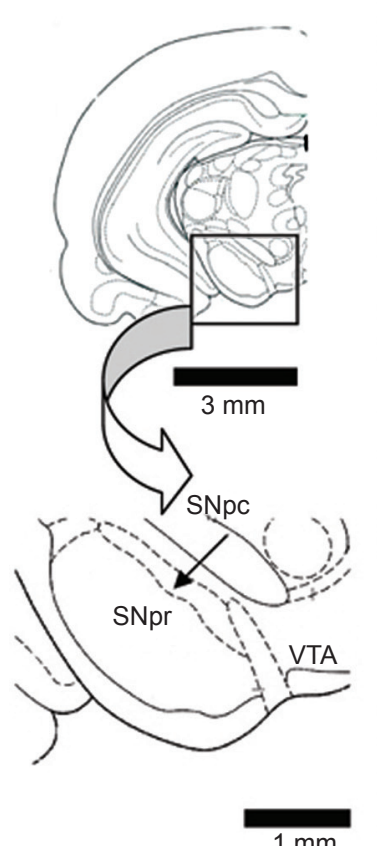

$1 \mathrm{~mm}$

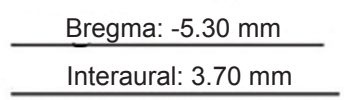

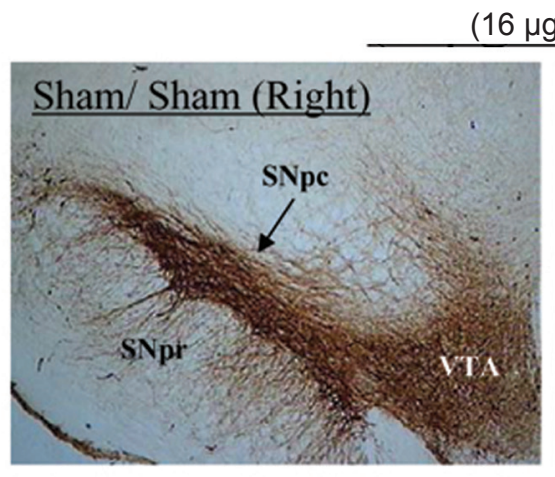
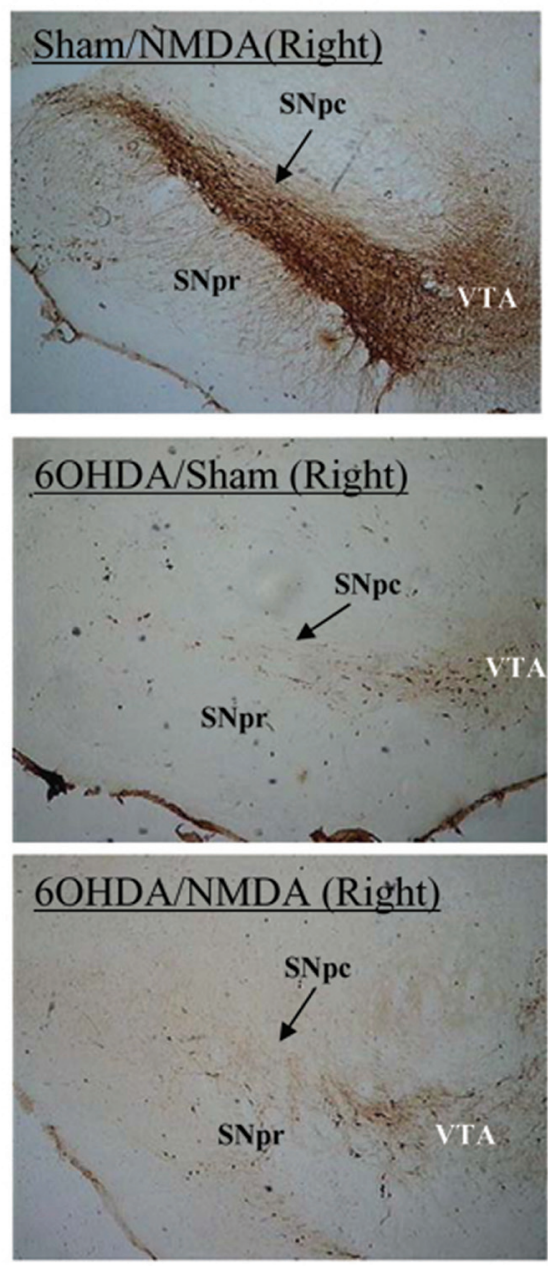
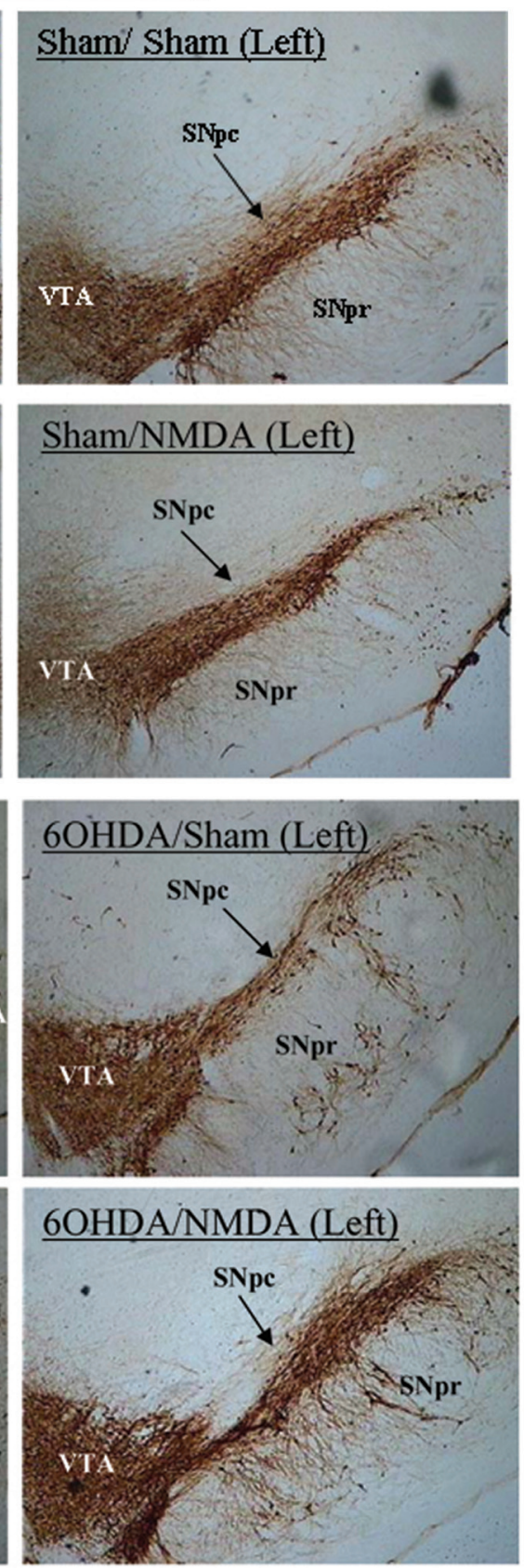

$1 \mathrm{~mm}$

Figure 5. Digitized images of TH immunohistochemistry in the SNpc and VTA after different treatments. Note decreased TH-immunoreactivity in the right SNpc and VTA in response to 6-OHDA injection into the right medial forebrain bundle. Photomicrographs represent TH immunostaining in the following groups: SNpc sham lesion/STN sham lesion (Sham/Sham), SNpc sham lesion/STN NMDA lesion (Sham/NMDA), SNpc 6-OHDA lesion/STN sham lesion (6-OHDA/Sham), and SNpc 6-OHDA lesion/STN NMDA lesion (6-OHDA/ NMDA). $\mathrm{SNpc}=$ substantia nigra pars compacta; SNpr = substantia nigra pars reticulata; STN = subthalamic nucleus; NMDA = Nmethyl-D-aspartate; 6-OHDA = 6-hydroxydopamine; VTA = ventral tegmental area. 

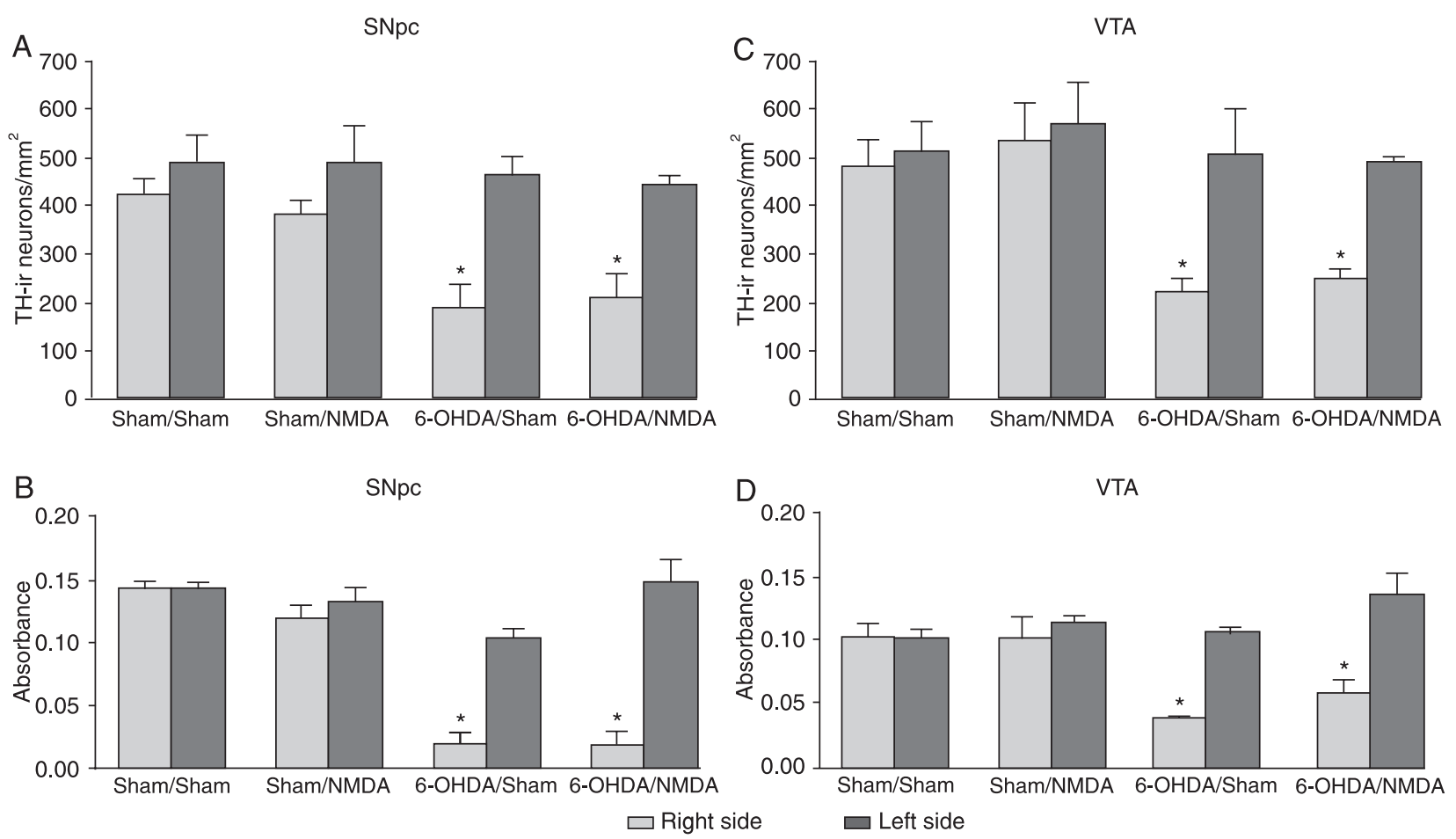

Figure 6. TH immunohistochemistry in the SNpc and VTA. $A$ and $C$, Tyrosine hydroxylase-immunoreactive (TH-ir) neuron density (neurons $/ \mathrm{mm}^{2}$ ). $B$ and $D$, TH immunostaining absorbance. Data are reported as means \pm SEM on the right and left sides for the following groups: SNpc sham lesion/STN sham lesion (Sham/Sham; $N=4)$, SNpc sham lesion/STN NMDA lesion (Sham/NMDA; $N=4)$, SNpc 6-OHDA lesion/STN sham lesion (6-OHDA/Sham; $N=4)$, and SNpc 6-OHDA lesion/STN NMDA lesion (6-OHDA/NMDA; $N=4)$. SNpc = substantia nigra pars compacta; STN = subthalamic nucleus; NMDA = N-methyl-D-aspartate; 6-OHDA = 6-hydroxydopamine; VTA = ventral tegmental area. ${ }^{*} \mathrm{P}<0.001$ compared to the Sham/Sham and Sham/NMDA groups (Duncan test after two-way ANOVA).

anesthesia following NMDA lesion of the STN (14).

\section{Discussion}

The mechanism responsible for the improvement of motor symptoms in PD after reversible or definitive inhibition of the STN is still unknown. In the present study, we determined whether STN lesion-induced motor recovery could be explained by the rescue of nigrostriatal dopaminergic neurons in a 6-OHDA-lesion rat model of PD. Our results show that STN lesion performed 15 days after 6-OHDA lesion of the nigrostriatal pathway reduced the apomorphine-induced rotational behavior. These data agree with previous reports that STN lesion reduces the number of apomorphine-induced contralateral rotations $(4,12,15)$. However, we found no improvement of hypokinesia in the open-field test. STN lesion has been shown to improve some, but not all, parkinsonian signs (14). Despite the lack of total recovery of motor function, STN lesion blocked the progression of motor impairment from the first to the second behavioral test in 6-OHDA-treated animals. Our experimental design was therefore effective in reproducing the anti-parkinsonian effects of STN lesion.
Our study did not detect an effect of STN lesion on the number of surviving TH-ir cells in the SNpc or VTA after 6-OHDA lesion of the MFB. Our results show a decrease of about $50 \%$ in the number of $\mathrm{TH}$-ir neurons per $\mathrm{mm}^{2}$ in the SNpc of animals treated with 6-OHDA and an apparently greater decrease in the absorbance measurements related to $\mathrm{TH}$ in the same groups. This apparent discrepancy can be explained by the fact that there is no linear correlation between neuronal density and absorbance measurements, because, regarding absorbance, the light absorbed by the tissue conforms to the Lambert-Beers law and is presented on a logarithmic scale, while the neuronal density is presented on an arithmetic scale $(26,30)$.

Our results showing that STN lesion did not affect the number of surviving TH-ir cells in the SNpc or VTA after 6-OHDA agree with the results of Bilbao et al. (20) who reported that subthalamotomy after 6-OHDA lesion did not promote immunohistochemical or electrophysiological changes in the nigrostriatal system. Luquin et al. (22) conducted a study on two groups of non-human primates, one subjected to STN lesion before MPTP administration and the other subjected to lesion after MPTP administration. In the second case, STN lesion produced an improvement of 
movements, but nigral degeneration was neither reduced nor prevented by prior STN lesion. Asimilar result was also obtained for monkeys undergoing subthalamotomy before MPTP.

An elegant study by Paul et al. (5) investigated the potential neuroprotective effects of STN lesion on SNpc cells lesioned with 6-OHDA two weeks later. Using fluorogold and $\mathrm{TH}$-immunoreactivity as markers of cell survival and dopaminergic phenotype, respectively, they showed that ablation of the STN led to a partial recovery of amphetamineinduced rotational behavior, but did not affect the number of fluorogold-ir cells, whereas it increased the number of $\mathrm{TH}$-ir cells. This suggests that STN lesion may have rescued the neurotransmitter phenotype in the remaining cells. In our study, if a recovery of impaired SNpc cells had occurred, increased striatal DA levels would be expected. Nevertheless, whereas 6-OHDA caused a decrease greater than $95 \%$ in striatal DA, DOPAC and HVA concentrations, the STN lesion had no effect on the concentrations of striatal DA and its metabolites. This means that, at least under the experimental conditions of the present study, the STN lesion neither preserved SNpc cells from death nor recruited the DA phenotype. Some studies using deep brain stimulation $(17,31)$ have shown enhanced DA or metabolite levels in the striatum, suggesting a mechanism whereby STN HFS improves motor symptoms in PD. However, the improvement of motor symptoms observed here cannot be explained by the increased release of DA in the striatum from residual dopaminergic neurons. Thus, it seems that the mechanism of action involved in the improvement of motor symptoms by STN lesion requires additional study. It is unlikely that the absence of dopaminergic protection was due to an incomplete lesion of the STN, because NMDA lesion was able to reverse the motor deficits induced by $6-\mathrm{OHDA}$ and to promote transitory contralateral hemiballism.

Bilbao et al. (20), studying the electrophysiology of SNpc neurons and the effects of subthalamotomy and levodopa treatment before 6-OHDA lesion, showed that $4 \mu \mathrm{g} 6$-OHDA caused a neuronal loss of almost $50 \%$ and that subthalamotomy did not modify the neuronal firing pattern or the reduction in TH-ir neurons. The 6-OHDAdose used in the present study $(16 \mu \mathrm{g})$ promoted an extensive loss of dopaminergic cells, approximately $57 \%$. This suggests that, in case of severe injury, when more than $50 \%$ of cells are lost, the STN lesion may not exert neuroprotection. Accordingly, Luquin et al. (22) showed that STN lesion did not protect the SNpc neurons against MPTP toxicity, promoting a $\mathrm{TH}$-ir cell death rate of over $80 \%$. In addition, Fang et al. (16) reported that in mild PD models, with less than $50 \%$ of neuronal loss, the inhibitory state is easily corrected by STN HFS, whereas in severe PD models (more than $70 \%$ of neuronal loss) the parkinsonian symptoms are more resistant to treatment.

Some investigators have reported that STN lesion prevented the loss of dopaminergic neurons in the SNpc after intrastriatal injection of 6-OHDA in rats $(13,32)$. However, the protection obtained following STN lesion was more effective when the lesion was performed before 6-OHDA administration rather than after it. Interestingly, it has been recently shown that intrapallidal injections of glutamate receptor agonists also promote suppressive effect on rotational behavior of 6-OHDA-lesioned rats, without altering dopaminergic degeneration (33).

Our data showed that motor deficits display an important improvement after STN lesion, indicating the existence of a more complex physiological network within the basal ganglia or the existence of other excitatory pathways that could be influenced by STN inactivation. Although numerous studies have suggested that the excitatory response observed in $\mathrm{SNpc}$ neurons is due to direct glutamatergic afferents from the STN (34), the pedunculopontine nucleus is the major source of glutamatergic input to SNpc neurons (35). Accordingly, Takada et al. (36) reported that pedunculopontine nucleus lesion attenuated the cell loss and parkinsonian motor deficits in MPTP-treated monkeys.

The glutamatergic projections from the STN alter the physiology of the globus pallidus (GPi), and changes in the neuronal activity of GPi have been shown in animal models of PD. Ni et al. (37), using electrophysiological recordings of GPi cells in rats with 6-OHDA lesion, concluded that the STN plays an important role in the modulation of GPi neuron activity, which might account for the therapeutic effect of STN lesion in PD.

Finally, another glutamatergic pathway to be considered is the STN projection to the striatum. A direct projection from the STN to the striatum, though less abundant (34), could account for a decreased glutamatergic activity in the striatum after STN lesion $(4,38)$. Supporting this idea, it has been reported that the beneficial effects of STN inactivation on parkinsonian symptoms are correlated with alteration in glutamatergic transmission in the striatum $(4,15,39)$, which is similar to the beneficial effects promoted by levodopa treatment. Moreover, STN lesion may also affect the activity of thalamo-cortical projections and thereby alter glutamate levels in the striatum. Accordingly, Luquin and Mitrofanis (40) have shown that previous removal of cortical inputs was able to protect SNpc cells against 6-OHDA lesion. Thus, the STN lesion-induced improvement in motor alterations might not be due to a direct effect of the lesion, but to an indirect effect on cortical/striatal activity.

Using the rescue protocol, which has clinical relevance, our data show that STN lesion is able to reverse motor deficits after severe 6-OHDA-induced lesion of the nigrostriatal pathway without rescuing dopaminergic neurons in the SNpc. Further studies are necessary to clarify the STN pathways involved in the improvement of motor symptoms in PD.

\section{Acknowledgments}

The authors would like to thank Ruither O.G. Carolino (PhD) for technical assistance. Research supported by CNPq, CAPES and FAPESP. 


\section{References}

1. Betarbet R, Sherer TB, Di Monte DA, Greenamyre JT. Mechanistic approaches to Parkinson's disease pathogenesis. Brain Pathol 2002; 12: 499-510.

2. Dauer W, Przedborski S. Parkinson's disease: mechanisms and models. Neuron 2003; 39: 889-909.

3. Przedborski S. Pathogenesis of nigral cell death in Parkinson's disease. Parkinsonism Relat Disord 2005; 11 (Suppl 1): S3-S7.

4. Centonze D, Gubellini P, Rossi S, Picconi B, Pisani A, Bernardi G, et al. Subthalamic nucleus lesion reverses motor abnormalities and striatal glutamatergic overactivity in experimental parkinsonism. Neuroscience 2005; 133: 831840.

5. Paul G, Meissner W, Rein S, Harnack D, Winter C, Hosmann $\mathrm{K}$, et al. Ablation of the subthalamic nucleus protects dopaminergic phenotype but not cell survival in a rat model of Parkinson's disease. Exp Neurol 2004; 185: 272-280.

6. Obeso JA, Rodriguez-Oroz MC, Rodriguez M, Lanciego JL, Artieda J, Gonzalo N, et al. Pathophysiology of the basal ganglia in Parkinson's disease. Trends Neurosci 2000; 23: S8-S19.

7. Temel Y, Visser-Vandewalle V, Kaplan S, Kozan R, Daemen MA, Blokland A, et al. Protection of nigral cell death by bilateral subthalamic nucleus stimulation. Brain Res 2006; 1120: 100-105.

8. Wang L, Kitai ST, Xiang Z. Modulation of excitatory synaptic transmission by endogenous glutamate acting on presynaptic group II mGluRs in rat substantia nigra compacta. $J$ Neurosci Res 2005; 82: 778-787.

9. Tir M, Devos D, Blond S, Touzet G, Reyns N, Duhamel A, et al. Exhaustive, one-year follow-up of subthalamic nucleus deep brain stimulation in a large, single-center cohort of parkinsonian patients. Neurosurgery 2007; 61: 297-304.

10. Benabid AL. Deep brain stimulation for Parkinson's disease. Curr Opin Neurobiol 2003; 13: 696-706.

11. Maesawa S, Kaneoke Y, Kajita Y, Usui N, Misawa N, Nakayama $A$, et al. Long-term stimulation of the subthalamic nucleus in hemiparkinsonian rats: neuroprotection of dopaminergic neurons. J Neurosurg 2004; 100: 679-687.

12. Blandini F, Garcia-Osuna M, Greenamyre JT. Subthalamic ablation reverses changes in basal ganglia oxidative metabolism and motor response to apomorphine induced by nigrostriatal lesion in rats. Eur J Neurosci 1997; 9: 14071413.

13. Carvalho GA, Nikkhah G. Subthalamic nucleus lesions are neuroprotective against terminal 6-OHDA-induced striatal lesions and restore postural balancing reactions. Exp Neurol 2001; 171: 405-417.

14. Henderson JM, Annett LE, Ryan LJ, Chiang W, Hidaka S, Torres EM, et al. Subthalamic nucleus lesions induce deficits as well as benefits in the hemiparkinsonian rat. Eur $\mathrm{J} \mathrm{Neu-}$ rosci 1999; 11: 2749-2757.

15. Touchon JC, Moore C, Frederickson J, Meshul CK. Lesion of subthalamic or motor thalamic nucleus in 6-hydroxydopamine-treated rats: effects on striatal glutamate and apomorphine-induced contralateral rotations. Synapse 2004; 51: 287-298.

16. Fang $\mathrm{X}$, Sugiyama $\mathrm{K}$, Akamine $\mathrm{S}$, Namba H. Improvements in motor behavioral tests during deep brain stimulation of the subthalamic nucleus in rats with different degrees of unilateral parkinsonism. Brain Res 2006; 1120: 202-210.

17. Lee KH, Blaha CD, Harris BT, Cooper S, Hitti FL, Leiter JC, et al. Dopamine efflux in the rat striatum evoked by electrical stimulation of the subthalamic nucleus: potential mechanism of action in Parkinson's disease. Eur J Neurosci 2006; 23: 1005-1014.

18. Armentero MT, Fancellu R, Nappi G, Bramanti $P$, Blandini F. Prolonged blockade of NMDA or mGluR5 glutamate receptors reduces nigrostriatal degeneration while inducing selective metabolic changes in the basal ganglia circuitry in a rodent model of Parkinson's disease. Neurobiol Dis 2006; 22: 1-9.

19. Phillips JM, Lam HA, Ackerson LC, Maidment NT. Blockade of mGluR glutamate receptors in the subthalamic nucleus ameliorates motor asymmetry in an animal model of Parkinson's disease. Eur J Neurosci 2006; 23: 151-160.

20. Bilbao G, Ruiz-Ortega JA, Miguens N, Ulibarri I, Linazasoro G, Gomez-Urquijo S, et al. Electrophysiological characterization of substantia nigra dopaminergic neurons in partially lesioned rats: effects of subthalamotomy and levodopa treatment. Brain Res 2006; 1084: 175-184.

21. Delfs JM, Ciaramitaro VM, Parry TJ, Chesselet MF. Subthalamic nucleus lesions: widespread effects on changes in gene expression induced by nigrostriatal dopamine depletion in rats. J Neurosci 1995; 15: 6562-6575.

22. Luquin MR, Saldise L, Guillen J, Belzunegui S, San Sebastian W, Izal A, et al. Does increased excitatory drive from the subthalamic nucleus contribute to dopaminergic neuronal death in Parkinson's disease? Exp Neurol 2006; 201: 407415.

23. Olanow CW, Jenner P, Tatton NA, Tatton WG. Neurodegeneration and Parkinson disease. In: Jankovik J, Tolosa $\mathrm{E}$ (Editors), Parkinson's disease and movement disorders. 3rd edn. Baltimore: Williams and Wikins; 1998. p 67-103.

24. Ferraz AC, Xavier LL, Hernandes S, Sulzbach M, Viola GG, Anselmo-Franci JA, et al. Failure of estrogen to protect the substantia nigra pars compacta of female rats from lesion induced by 6-hydroxydopamine. Brain Res 2003; 986: 200205.

25. Ferraz AC, Matheussi F, Szawka RE, Rizelio V, Delattre AM, Rigon $\mathrm{P}$, et al. Evaluation of estrogen neuroprotective effect on nigrostriatal dopaminergic neurons following 6-hydroxydopamine injection into the substantia nigra pars compacta or the medial forebrain bundle. Neurochem Res 2008; 33: 1238-1246.

26. Paxinos G, Watson C. The rat brain in stereotaxic coordinates. San Diego: Academic Press; 1997.

27. Naliwaiko K, Araujo RL, da Fonseca RV, Castilho JC, Andreatini R, Bellissimo MI, et al. Effects of fish oil on the central nervous system: a new potential antidepressant? Nutr Neurosci 2004; 7: 91-99.

28. Xavier LL, Viola GG, Ferraz AC, Da Cunha C, Deonizio JM, Netto CA, et al. A simple and fast densitometric method for the analysis of tyrosine hydroxylase immunoreactivity in the substantia nigra pars compacta and in the ventral tegmental area. Brain Res Brain Res Protoc 2005; 16: 58-64.

29. Xue YQ, Zhao LR, Guo WP, Duan WM. Intrastriatal administration of erythropoietin protects dopaminergic neurons 
and improves neurobehavioral outcome in a rat model of Parkinson's disease. Neuroscience 2007; 146: 1245-1258.

30. Chieco P, Jonker A, Melchiorri C, Vanni G, Van Noorden CJ. A user's guide for avoiding errors in absorbance image cytometry: a review with original experimental observations. Histochem J 1994; 26: 1-19.

31. Meissner W, Harnack D, Paul G, Reum T, Sohr R, Morgenstern $R$, et al. Deep brain stimulation of subthalamic neurons increases striatal dopamine metabolism and induces contralateral circling in freely moving 6-hydroxydopaminelesioned rats. Neurosci Lett 2002; 328: 105-108.

32. Benabid AL, Piallat B, Wallace B, Benazzouza A, Benazzouza $A$, Lenartzb $D$, et al. Might deep brain stimulation of the subthalamic nucleus be neuroprotective in patients with Parkinson's disease? Thalamus Relat Syst 2003; 2: 95-102.

33. Agari T, Yasuhara T, Matsui T, Kuramoto S, Kondo A, Miyoshi Y, et al. Intrapallidal metabotropic glutamate receptor activation in a rat model of Parkinson's disease: behavioral and histological analyses. Brain Res 2008; 1203: 189-196.

34. Parent A, Hazrati LN. Functional anatomy of the basal ganglia. II. The place of subthalamic nucleus and external pallidum in basal ganglia circuitry. Brain Res Brain Res Rev 1995; 20: 128-154.

35. Bezard E, Gross CE. Compensatory mechanisms in ex- perimental and human parkinsonism: towards a dynamic approach. Prog Neurobiol 1998; 55: 93-116.

36. Takada M, Matsumura M, Kojima J, Yamaji Y, Inase M, Tokuno $\mathrm{H}$, et al. Protection against dopaminergic nigrostriatal cell death by excitatory input ablation. Eur $\mathrm{J}$ Neurosci 2000; 12: 1771-1780.

37. Ni Z, Bouali-Benazzouz R, Gao D, Benabid AL, Benazzouz A. Changes in the firing pattern of globus pallidus neurons after the degeneration of nigrostriatal pathway are mediated by the subthalamic nucleus in the rat. Eur J Neurosci 2000; 12: 4338-4344.

38. Centonze D, Rossi S, Gubellini P, De Chiara V, Tscherter A, Prosperetti $\mathrm{C}$, et al. Deficits of glutamate transmission in the striatum of experimental hemiballism. Neuroscience 2006; 143: 213-221.

39. Lee KH, Kristic K, van Hoff R, Hitti FL, Blaha C, Harris B, et al. High-frequency stimulation of the subthalamic nucleus increases glutamate in the subthalamic nucleus of rats as demonstrated by in vivo enzyme-linked glutamate sensor. Brain Res 2007; 1162: 121-129.

40. Luquin N, Mitrofanis J. Does the cerebral cortex exacerbate dopaminergic cell death in the substantia nigra of 6OHDAlesioned rats? Parkinsonism Relat Disord 2008; 14: 213223. 